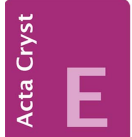
COMMUNICATIONS

ISSN 2056-9890

\section{Crystal structure of tris(3-methyl-1H- pyrazol-1-yl)methane}

\author{
Margaret A. Goodman, ${ }^{a}$ M. Scott Goodman, ${ }^{\text {b }}$ \\ Alexander Y. Nazarenko ${ }^{\mathrm{b}}$ and Ealin N. Patel ${ }^{\mathrm{b}}$ \\ aChemistry Department, D'Youville College, 320 Porter Avenue, Buffalo, NY 14201, \\ USA, and ${ }^{\mathbf{b}}$ Chemistry Department, SUNY Buffalo State, 1300 Elmwood Ave, Buffalo, \\ NY 14222, USA. *Correspondence e-mail: nazareay@buffalostate.edu
}

Received 12 September 2015; accepted 15 September 2015

Edited by M. Zeller, Youngstown State University, USA

The title molecule, $\mathrm{C}_{13} \mathrm{H}_{16} \mathrm{~N}_{6}$, crystallizes from hexane as a molecular crystal with no strong intermolecular interactions (the shortest $\mathrm{C}-\mathrm{H} \cdots \mathrm{N}$ contact is longer than $3.38 \AA$ ). A relatively short intramolecular contact $(3.09 \AA)$ has a $\mathrm{C}-$ $\mathrm{H} \cdots \mathrm{N}$ angle of $118^{\circ}$ which is quite small to be still considered a hydrogen bond. The three pyrazole rings form a propellerlike motif, with one methylpyrazole unit almost perpendicular to the mean plane of the three rings $\left[82.20(6)^{\circ}\right]$. The other two methylpyrazole units, with nitrogen donor atoms oriented in opposite directions, are oriented at $67.26(6)$ and $72.53(6)^{\circ}$ to the mean plane.

Keywords: crystal structure; $1,1^{\prime}, 1^{\prime \prime}$-methanetriyltris(3-methyl-1H-pyrazole); tripyrazolylmethane.

CCDC reference: 1424633

\section{Related literature}

For syntheses and reactions of trispyrazolylmethanes and their complexes with transition metals, see: Goodman et al. (2012); Jameson \& Castellano (1998); Reger et al. (2000).<smiles>Cc1ccn(C(n2ccc(C)n2)n2ccc(C)n2)n1</smiles>

\section{Experimental}

2.1. Crystal data

$\mathrm{C}_{13} \mathrm{H}_{16} \mathrm{~N}_{6}$

$M_{r}=256.32$

Monoclinic, $P 2_{1} / c$

$a=12.0881(8) \AA$

$b=13.4178(10) \AA$

$c=9.0985(6) \AA$

$\beta=111.630(2)^{\circ}$

$$
\begin{aligned}
& V=1371.82(16) \AA^{3} \\
& Z=4 \\
& \text { Mo } K \alpha \text { radiation } \\
& \mu=0.08 \mathrm{~mm}^{-1} \\
& T=173 \mathrm{~K} \\
& 0.60 \times 0.48 \times 0.29 \mathrm{~mm}
\end{aligned}
$$

\subsection{Data collection}

Bruker Photon-100 CMOS diffractometer

Absorption correction: multi-scan (SADABS; Bruker, 2014) $T_{\min }=0.706, T_{\max }=0.747$

22036 measured reflections 2612 independent reflections 2171 reflections with $I>2 \sigma(I)$ $R_{\text {int }}=0.029$

\subsection{Refinement}

$R\left[F^{2}>2 \sigma\left(F^{2}\right)\right]=0.037$

$w R\left(F^{2}\right)=0.099$

$S=1.05$

2612 reflections

225 parameters

$\mathrm{H}$ atoms treated by a mixture of independent and constrained refinement

$\Delta \rho_{\max }=0.28$ e $\AA^{-3}$

$\Delta \rho_{\min }=-0.19$ e $\AA^{-3}$

Data collection: APEX2 (Bruker, 2013); cell refinement: SAINT (Bruker, 2013); data reduction: $S A I N T$; $\operatorname{program}(\mathrm{s})$ used to solve structure: SHELXT (Sheldrick, 2015a); program(s) used to refine structure: SHELXL2014 (Sheldrick, 2015b); molecular graphics: OLEX2 (Dolomanov et al., 2009); software used to prepare material for publication: $O L E X 2$.

\section{Acknowledgements}

Financial support from the State University of New York for acquisition and maintenance of the $\mathrm{X}$-ray diffractometer is gratefully acknowledged.

Supporting information for this paper is available from the IUCr electronic archives (Reference: ZL2643).

\section{References}

Bruker (2013). APEX2 and SAINT. Bruker AXS Inc., Madison, Wisconsin, USA.

Bruker (2014). SADABS. Bruker AXS Inc., Madison, Wisconsin, USA.

Dolomanov, O. V., Bourhis, L. J., Gildea, R. J., Howard, J. A. K. \& Puschmann, H. (2009). J. Appl. Cryst. 42, 339-341.

Goodman, M. A., Nazarenko, A. Y., Casavant, B. J., Li, Z., Brennessel, W. W., DeMarco, M. J., Long, G. \& Goodman, M. S. (2012). Inorg. Chem. 51, 10841093.

Jameson, D. L. \& Castellano, R. K. (1998). Inorg. Synth. 32, 51-63.

Reger, D. L., Grattan, T. C., Brown, K. J., Little, C. A., Lamba, J. J. S., Rheingold, A. L. \& Sommer, R. D. (2000). J. Organomet. Chem. 607, 120128.

Sheldrick, G. M. (2015a). Acta Cryst. A71, 3-8.

Sheldrick, G. M. (2015b). Acta Cryst. C71, 3-8. 


\section{supporting information}

Acta Cryst. (2015). E71, o816 [https://doi.org/10.1107/S2056989015017247]

\section{Crystal structure of tris(3-methyl-1H-pyrazol-1-yl)methane}

\section{Margaret A. Goodman, M. Scott Goodman, Alexander Y. Nazarenko and Ealin N. Patel}

\section{S1. Chemical context}

This report is part of our continious effort to study substituted trispyrazolylmethanes and their complexes with various metal ions. Because all synthetic procedures yield a complex mixture of isomers, positive identification of the ligand molecule by X-ray diffractometry is essential for future research.

\section{S2. Refinement}

Crystal data, data collection and structure refinement details are summarized in Table 1.

All hydrogen atoms were located in electron difference density Fourier maps and were refined in an isotropic approximation. One methyl group (C5) was treated as disordered (SHELXL instruction AFIX 124). Isotropic parameters of atoms $\mathrm{H} 1$ and of disordered methyl group hydrogen atoms were constrained as $\mathrm{U}_{\mathrm{H}}=1.2 \mathrm{U}_{\mathrm{C}}$.

Reflections 100 and 110 were too close to the beamstop to be measured reliably and were excluded from refinement.

\section{S3. Synthesis and crystallization}

Following the general method of Reger et al. (2000), 3-methylpyrazole ( $6.0 \mathrm{~mL}, 75.0 \mathrm{mmol})$, tetrabutylammonium bromide $(1.21 \mathrm{~g}, 3.75 \mathrm{mmol})$, and sodium carbonate $(47.0 \mathrm{~g}, 0.45 \mathrm{~mol})$ were heated together in a biphasic mixture of deionized water $(75 \mathrm{~mL})$ and chloroform $(40 \mathrm{~mL})$. The reaction mixture was allowed to gently reflux for approximately 72 hours under argon. After cooling and filtering, the organic layer was separated from the aqueous layer. The aqueous layer was extracted three times with diethyl ether $(100 \mathrm{~mL})$, and the combined organic portions were washed twice with $100 \mathrm{~mL}$ portions of $\mathrm{H}_{2} \mathrm{O}$. The organic mixture was dried $\left(\mathrm{Na}_{2} \mathrm{SO}_{4}\right)$ and the solvents were removed under vacuum to give a dark, brown oil. ${ }^{1} \mathrm{H}$ NMR analysis showed this to be mainly a mixture of all four regioisomers of the tris(pyrazolyl)methanes derived from 3-methylpyrazole.

The crude mixture of tris(pyrazolyl)methane regioisomers was first isomerized according to the method of Jameson \& Castellano (1998). The crude brown oil (1.0 g) was combined with a catalytic amount of $p$-toluenesulfonic acid $(0.060 \mathrm{~g})$ and a small amount $(50 \mu \mathrm{L})$ of 3-methylpyrazole and heated at reflux in dry toluene $(15 \mathrm{~mL})$ for 24 hours under argon. After cooling, the mixture was washed twice with $100 \mathrm{~mL}$ portions of saturated $\mathrm{NaHCO}_{3}(\mathrm{aq})$. The aqueous extracts were then extracted once with $\mathrm{CH}_{2} \mathrm{Cl}_{2}(100 \mathrm{~mL})$. The organic layers were combined, dried with $\mathrm{Na}_{2} \mathrm{SO}_{4}$, and evaporated under reduced pressure to give a dark yellow oil. NMR analysis of this oil showed that it contained a 2:1 mixture of the desired tris(pyrazolyl)methane and another regioisomer.

For purification, the material was dissolved in a minimum amount of hot hexane and allowed to crystallize at room temperature for 24 hours. The resulting yellow/brown crystals were separated under a microscope. The larger, clear, and darker-colored lozenges were separated from the smaller, opaque, and lighter plates. These smaller crystals tend to form in masses, often growing on the larger crystals and the bottom of the flask. The larger crystals were scraped clean of as much of the other material as possible under the microscope. The large crystals separated in this fashion were typically at 
least $85 \%$ of target compound. This material was then carefully crystallized from hot hexanes after decolorization with carbon in the same solvent.

A suitable crystal was carefully cut from a larger block. A bigger crystal demonstrated the same structure in a preliminary X-ray experiment.

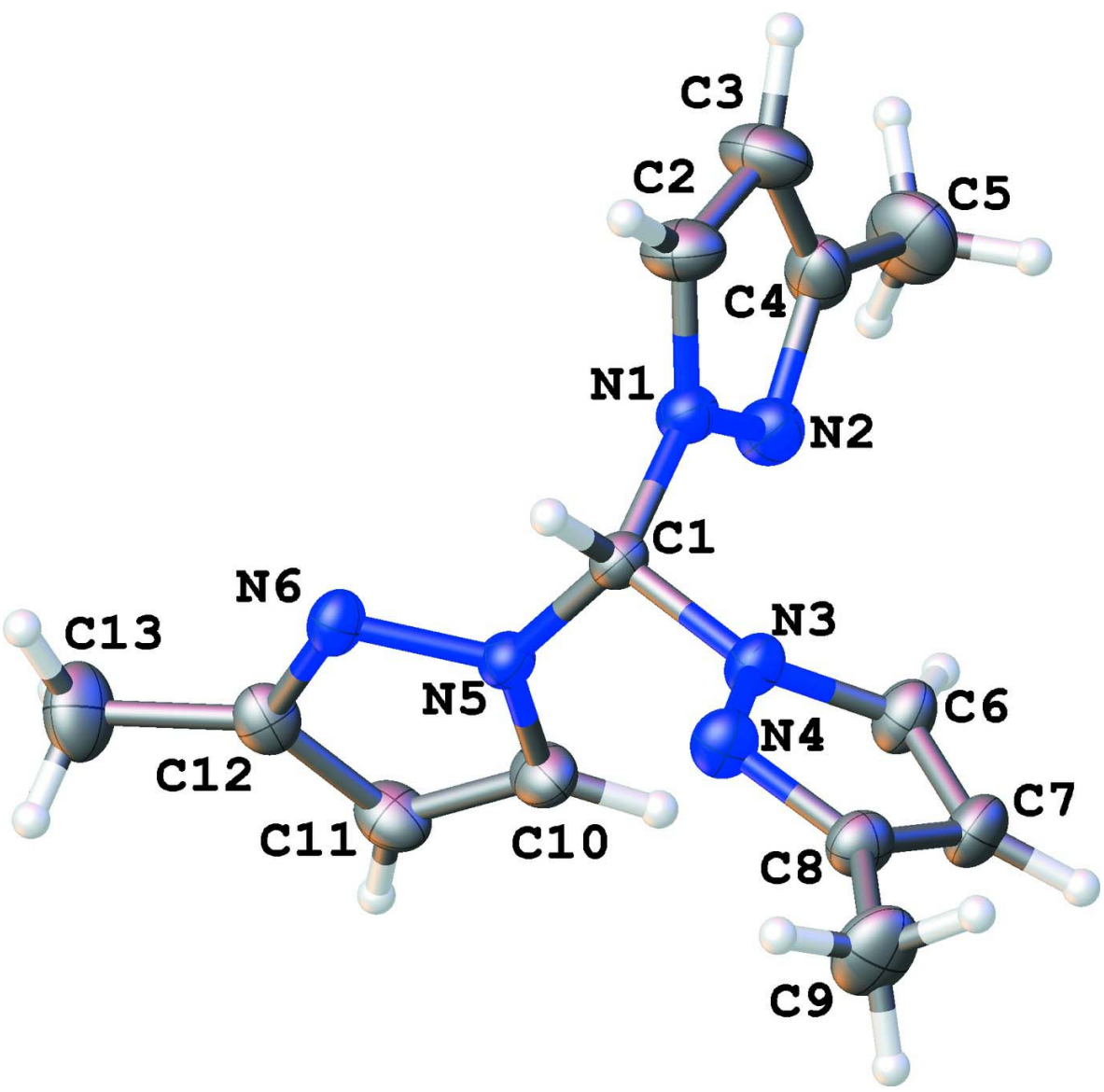

Figure 1

The molecular structure of the title compound. Displacement elipsoids are drawn at the $50 \%$ probability level. Disorder of $\mathrm{H}$ atoms bonded to $\mathrm{C} 5$ are omitted for clarity. 


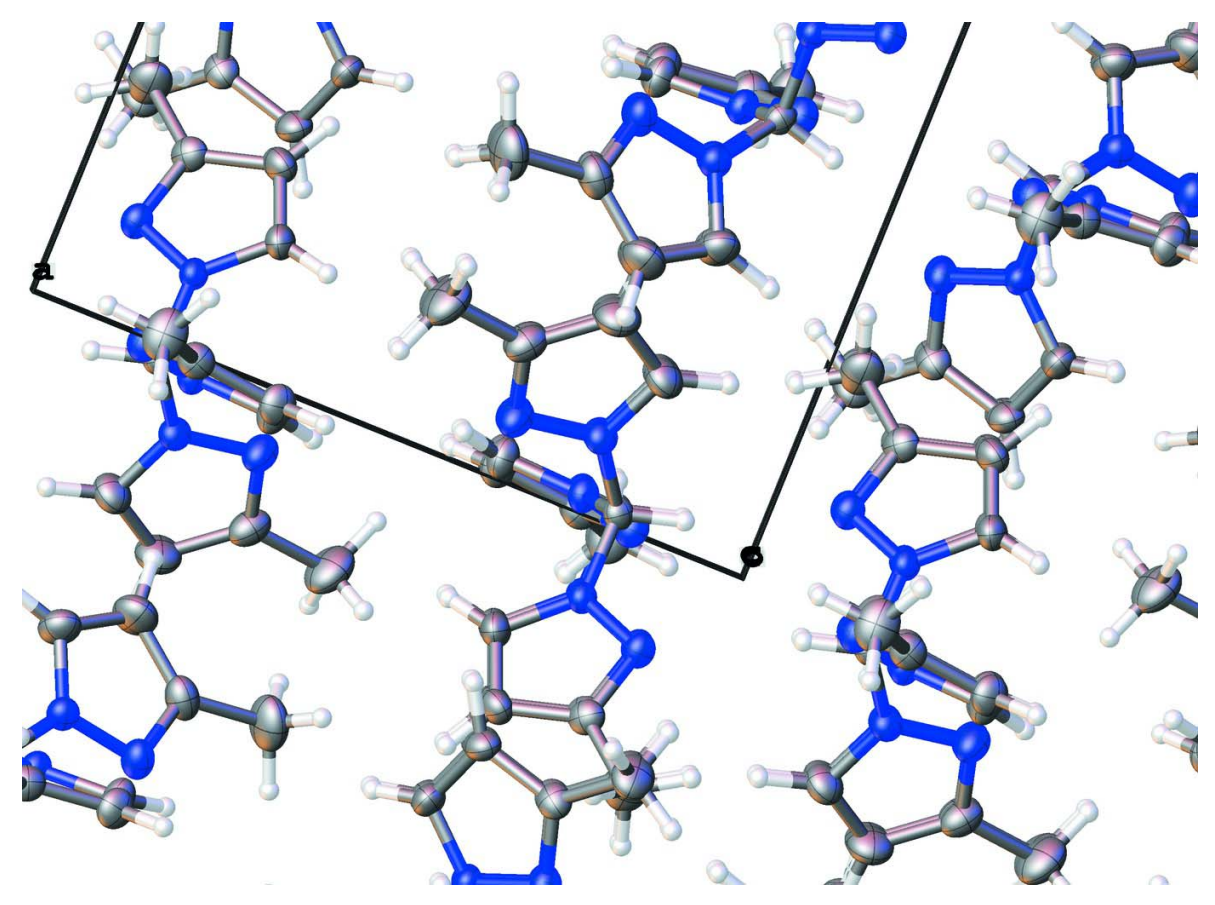

Figure 2

Packing diagram of the title molecule. View along the $c$ axis.

1,1',1'-Methanetriyltris(3-methyl-1H-pyrazole)

Crystal data

$\mathrm{C}_{13} \mathrm{H}_{16} \mathrm{~N}_{6}$

$M_{r}=256.32$

Monoclinic, $P 2{ }_{1} / c$

$a=12.0881(8) \AA$

$b=13.4178(10) \AA$

$c=9.0985(6) \AA$

$\beta=111.630(2)^{\circ}$

$V=1371.82(16) \AA^{3}$

$Z=4$

$F(000)=544$

$D_{\mathrm{x}}=1.241 \mathrm{Mg} \mathrm{m}^{-3}$

Mo $K \alpha$ radiation, $\lambda=0.71073 \AA$

Cell parameters from 9433 reflections

$\theta=2.9-25.7^{\circ}$

$\mu=0.08 \mathrm{~mm}^{-1}$

$T=173 \mathrm{~K}$

Block, colourless

$0.60 \times 0.48 \times 0.29 \mathrm{~mm}$

\section{Data collection}

Bruker Photon-100 CMOS diffractometer

Radiation source: sealedtube $\varphi$ and $\omega$ scans

Absorption correction: multi-scan

(SADABS; Bruker, 2014)

$T_{\min }=0.706, T_{\max }=0.747$

22036 measured reflections

\section{Refinement}

Refinement on $F^{2}$

Least-squares matrix: full

$R\left[F^{2}>2 \sigma\left(F^{2}\right)\right]=0.037$

$w R\left(F^{2}\right)=0.099$

$S=1.05$

2612 independent reflections

2171 reflections with $I>2 \sigma(I)$

$R_{\text {int }}=0.029$

$\theta_{\text {max }}=25.7^{\circ}, \theta_{\min }=2.9^{\circ}$

$h=-14 \rightarrow 14$

$k=-16 \rightarrow 16$

$l=-10 \rightarrow 11$

2612 reflections

225 parameters

0 restraints

Hydrogen site location: mixed 
$\mathrm{H}$ atoms treated by a mixture of independent and constrained refinement

$w=1 /\left[\sigma^{2}\left(F_{\mathrm{o}}^{2}\right)+(0.0457 P)^{2}+0.5131 P\right]$

where $P=\left(F_{\mathrm{o}}^{2}+2 F_{\mathrm{c}}^{2}\right) / 3$

$$
\begin{aligned}
& (\Delta / \sigma)_{\max }<0.001 \\
& \Delta \rho_{\max }=0.28 \mathrm{e} \AA^{-3} \\
& \Delta \rho_{\min }=-0.19 \mathrm{e} \AA^{-3}
\end{aligned}
$$

\section{Special details}

Experimental. SADABS-2014/5 (Bruker,2014/5) was used for absorption correction. wR2(int) was 0.0499 before and 0.0468 after correction. The ratio of minimum to maximum transmission is 0.9453 . The $\lambda / 2$ correction factor is 0.00150 .

Geometry. All esds (except the esd in the dihedral angle between two 1.s. planes) are estimated using the full covariance matrix. The cell esds are taken into account individually in the estimation of esds in distances, angles and torsion angles; correlations between esds in cell parameters are only used when they are defined by crystal symmetry. An approximate (isotropic) treatment of cell esds is used for estimating esds involving l.s. planes.

Refinement. 1. Fixed Uiso At 1.2 times of: All C(H) groups, All C(H,H,H,H,H,H) groups 2. Others $\operatorname{Sof}(\mathrm{H} 5 \mathrm{D})=\operatorname{Sof}(\mathrm{H} 5 \mathrm{E})=\operatorname{Sof}(\mathrm{H} 5 \mathrm{~F})=1-\mathrm{FVAR}(1) \operatorname{Sof}(\mathrm{H} 5 \mathrm{~A})=\operatorname{Sof}(\mathrm{H} 5 \mathrm{~B})=\operatorname{Sof}(\mathrm{H} 5 \mathrm{C})=$ FVAR(1) 3.a Disordered Me refined with

\begin{tabular}{|c|c|c|c|c|c|}
\hline & $x$ & $y$ & $z$ & $U_{\text {iso }} * / U_{\text {eq }}$ & Occ. $(<1)$ \\
\hline N1 & $0.75721(10)$ & $0.58635(9)$ & $0.89470(13)$ & $0.0257(3)$ & \\
\hline $\mathrm{C} 1$ & $0.81554(12)$ & $0.51681(10)$ & $0.82664(15)$ & $0.0231(3)$ & \\
\hline H1 & $0.8972(14)$ & $0.5366(11)$ & 0.8603 (18) & $0.028^{*}$ & \\
\hline $\mathrm{N} 2$ & $0.64449(11)$ & $0.57102(10)$ & $0.88717(14)$ & 0.0322 & \\
\hline $\mathrm{C} 2$ & $0.80352(15)$ & $0.67251(12)$ & $0.96750(19)$ & $0.0379(4)$ & \\
\hline $\mathrm{H} 2$ & $0.8860(18)$ & $0.6925(14)$ & $0.984(2)$ & $0.051(5)^{*}$ & \\
\hline N3 & $0.76487(10)$ & $0.52045(9)$ & $0.65612(13)$ & $0.0247(3)$ & \\
\hline $\mathrm{C} 3$ & $0.71772(17)$ & $0.71542(14)$ & $1.0092(2)$ & $0.0469(4)$ & \\
\hline H3 & $0.7246(19)$ & 0.7797 (18) & $1.066(3)$ & $0.074(7)^{*}$ & \\
\hline N4 & $0.83993(11)$ & $0.50592(9)$ & $0.57737(14)$ & $0.0288(3)$ & \\
\hline $\mathrm{C} 4$ & $0.62038(14)$ & $0.65090(12)$ & $0.95767(18)$ & $0.0358(4)$ & \\
\hline N5 & $0.81296(9)$ & $0.41679(8)$ & $0.88404(12)$ & $0.0224(3)$ & \\
\hline $\mathrm{C} 5$ & $0.50296(17)$ & $0.66100(16)$ & $0.9753(2)$ & $0.0547(5)$ & \\
\hline H5A & $0.50076(17)$ & $0.7279(9)$ & $1.0330(8)$ & $0.066^{*}$ & 0.544 (19) \\
\hline H5B & $0.4350(9)$ & $0.66087(16)$ & $0.8637(14)$ & $0.066^{*}$ & 0.544 (19) \\
\hline $\mathrm{H} 5 \mathrm{C}$ & $0.4909(2)$ & $0.6013(8)$ & $1.0416(9)$ & $0.066^{*}$ & 0.544 (19) \\
\hline H5D & $0.4504(7)$ & $0.5989(8)$ & $0.9259(7)$ & $0.066^{*}$ & $0.456(19)$ \\
\hline H5E & $0.5161(2)$ & $0.66585(18)$ & $1.0952(15)$ & $0.066^{*}$ & $0.456(19)$ \\
\hline $\mathrm{H} 5 \mathrm{~F}$ & $0.4602(6)$ & $0.7254(8)$ & $0.9172(8)$ & $0.066^{*}$ & $0.456(19)$ \\
\hline N6 & $0.90717(10)$ & $0.38708(9)$ & $1.01297(12)$ & 0.0249 & \\
\hline C6 & $0.64987(13)$ & $0.52449(12)$ & $0.55706(17)$ & 0.0310 & \\
\hline H6 & $0.5895(15)$ & $0.5348(12)$ & 0.5961 (19) & $0.031(4)^{*}$ & \\
\hline $\mathrm{C} 7$ & $0.64953(14)$ & $0.51349(12)$ & $0.40812(17)$ & $0.0332(4)$ & \\
\hline $\mathrm{H} 7$ & $0.5830(16)$ & $0.5140(13)$ & $0.313(2)$ & $0.043(5)^{*}$ & \\
\hline $\mathrm{C} 8$ & $0.76915(13)$ & $0.50248(11)$ & $0.42596(16)$ & 0.0299 (3) & \\
\hline C9 & $0.82000(19)$ & $0.48816(18)$ & $0.3011(2)$ & $0.0473(5)$ & \\
\hline H9A & $0.905(2)$ & $0.4833(17)$ & $0.347(3)$ & $0.072(7)^{*}$ & \\
\hline H9B & $0.784(2)$ & $0.433(2)$ & $0.236(3)$ & $0.084(8)^{*}$ & \\
\hline $\mathrm{H} 9 \mathrm{C}$ & $0.805(2)$ & $0.548(2)$ & $0.234(3)$ & $0.088(8)^{*}$ & \\
\hline $\mathrm{C} 10$ & $0.72423(13)$ & $0.34908(11)$ & $0.84262(18)$ & $0.0311(3)$ & \\
\hline
\end{tabular}
riding coordinates and stretchable bonds: $\mathrm{C} 5(\mathrm{H} 5 \mathrm{~A}, \mathrm{H} 5 \mathrm{~B}, \mathrm{H} 5 \mathrm{C}, \mathrm{H} 5 \mathrm{D}, \mathrm{H} 5 \mathrm{E}, \mathrm{H} 5 \mathrm{~F})$

Fractional atomic coordinates and isotropic or equivalent isotropic displacement parameters $\left(\AA^{2}\right)$ 


\begin{tabular}{lllll} 
H10 & $0.6507(15)$ & $0.3624(12)$ & $0.755(2)$ & $0.036(4)^{*}$ \\
C11 & $0.76199(14)$ & $0.27160(12)$ & $0.94568(19)$ & $0.0332(4)$ \\
H11 & $0.7205(15)$ & $0.2122(13)$ & $0.945(2)$ & $0.039(5)^{*}$ \\
C12 & $0.87610(13)$ & $0.29819(11)$ & $1.04977(16)$ & $0.0285(3)$ \\
C13 & $0.95886(18)$ & $0.24190(16)$ & $1.1873(2)$ & $0.0454(4)$ \\
H13A & $1.032(2)$ & $0.2793(19)$ & $1.244(3)$ & $0.086(8)^{*}$ \\
H13B & $0.921(2)$ & $0.2225(19)$ & $1.253(3)$ & $0.088(8)^{*}$ \\
H13C & $0.982(2)$ & $0.183(2)$ & $1.150(3)$ & $0.094(9)^{*}$ \\
\hline
\end{tabular}

Atomic displacement parameters $\left(\AA^{2}\right)$

\begin{tabular}{lllllll}
\hline & $U^{11}$ & $U^{22}$ & $U^{33}$ & $U^{12}$ & $U^{13}$ & $U^{23}$ \\
\hline $\mathrm{N} 1$ & $0.0252(6)$ & $0.0268(6)$ & $0.0242(6)$ & $-0.0002(5)$ & $0.0081(5)$ & $-0.0029(5)$ \\
$\mathrm{C} 1$ & $0.0219(7)$ & $0.0251(7)$ & $0.0210(7)$ & $-0.0020(5)$ & $0.0063(5)$ & $-0.0013(5)$ \\
$\mathrm{N} 2$ & $0.0272(7)$ & $0.0379(7)$ & $0.0315(7)$ & $0.0033(5)$ & $0.0108(5)$ & $-0.0050(5)$ \\
$\mathrm{C} 2$ & $0.0404(10)$ & $0.0334(9)$ & $0.0411(9)$ & $-0.0069(7)$ & $0.0163(7)$ & $-0.0108(7)$ \\
$\mathrm{N} 3$ & $0.0224(6)$ & $0.0305(7)$ & $0.0209(6)$ & $-0.0011(5)$ & $0.0077(5)$ & $-0.0003(5)$ \\
$\mathrm{C} 3$ & $0.0528(11)$ & $0.0350(10)$ & $0.0560(11)$ & $0.0016(8)$ & $0.0237(9)$ & $-0.0134(8)$ \\
$\mathrm{N} 4$ & $0.0281(7)$ & $0.0350(7)$ & $0.0261(6)$ & $0.0001(5)$ & $0.0134(5)$ & $0.0006(5)$ \\
C4 & $0.0351(9)$ & $0.0406(9)$ & $0.0316(8)$ & $0.0104(7)$ & $0.0122(7)$ & $0.0000(7)$ \\
N5 & $0.0199(6)$ & $0.0241(6)$ & $0.0221(6)$ & $-0.0007(4)$ & $0.0064(4)$ & $-0.0015(5)$ \\
C5 & $0.0450(11)$ & $0.0647(13)$ & $0.0602(12)$ & $0.0151(9)$ & $0.0260(9)$ & $-0.0059(10)$ \\
N6 & $0.0231(6)$ & $0.0310(7)$ & $0.0207(6)$ & $0.0004(5)$ & $0.0081(5)$ & $0.0023(5)$ \\
C6 & $0.0236(7)$ & $0.0424(9)$ & $0.0252(7)$ & $-0.0006(6)$ & $0.0070(6)$ & $-0.0003(6)$ \\
C7 & $0.0317(8)$ & $0.0409(9)$ & $0.0224(7)$ & $-0.0006(7)$ & $0.0048(6)$ & $0.0004(6)$ \\
C8 & $0.0347(8)$ & $0.0309(8)$ & $0.0245(7)$ & $-0.0015(6)$ & $0.0115(6)$ & $0.0007(6)$ \\
C9 & $0.0467(11)$ & $0.0697(14)$ & $0.0302(9)$ & $-0.0003(10)$ & $0.0195(8)$ & $-0.0020(9)$ \\
C10 & $0.0236(8)$ & $0.0287(8)$ & $0.0374(8)$ & $-0.0033(6)$ & $0.0069(7)$ & $-0.0032(6)$ \\
C11 & $0.0314(8)$ & $0.0262(8)$ & $0.0455(9)$ & $-0.0045(6)$ & $0.0182(7)$ & $0.0001(7)$ \\
C12 & $0.0302(8)$ & $0.0309(8)$ & $0.0295(7)$ & $0.0018(6)$ & $0.0169(6)$ & $0.0040(6)$ \\
C13 & $0.0448(11)$ & $0.0479(11)$ & $0.0436(10)$ & $0.0026(9)$ & $0.0163(9)$ & $0.0205(9)$ \\
& & & & & &
\end{tabular}

Geometric parameters $\left(A,{ }^{\circ}\right)$

\begin{tabular}{llll}
\hline $\mathrm{N} 1-\mathrm{C} 1$ & $1.4398(18)$ & $\mathrm{C} 5-\mathrm{H} 5 \mathrm{D}$ & $1.045(13)$ \\
$\mathrm{N} 1-\mathrm{N} 2$ & $1.3546(17)$ & $\mathrm{C} 5-\mathrm{H} 5 \mathrm{E}$ & $1.045(13)$ \\
$\mathrm{N} 1-\mathrm{C} 2$ & $1.347(2)$ & $\mathrm{C} 5-\mathrm{H} 5 \mathrm{~F}$ & $1.045(13)$ \\
$\mathrm{C} 1-\mathrm{H} 1$ & $0.956(16)$ & $\mathrm{N} 6-\mathrm{C} 12$ & $1.3298(19)$ \\
$\mathrm{C} 1-\mathrm{N} 3$ & $1.4436(17)$ & $\mathrm{C} 6-\mathrm{H} 6$ & $0.932(17)$ \\
$\mathrm{C} 1-\mathrm{N} 5$ & $1.4446(17)$ & $\mathrm{C} 6-\mathrm{C} 7$ & $1.362(2)$ \\
$\mathrm{N} 2-\mathrm{C} 4$ & $1.3353(19)$ & $\mathrm{C} 7-\mathrm{H} 7$ & $0.939(18)$ \\
$\mathrm{C} 2-\mathrm{H} 2$ & $0.99(2)$ & $\mathrm{C} 7-\mathrm{C} 8$ & $1.402(2)$ \\
$\mathrm{C} 2-\mathrm{C} 3$ & $1.357(2)$ & $\mathrm{C} 8-\mathrm{C} 9$ & $1.490(2)$ \\
$\mathrm{N} 3-\mathrm{N} 4$ & $1.3615(16)$ & $\mathrm{C} 9-\mathrm{H} 9 \mathrm{~A}$ & $0.96(2)$ \\
$\mathrm{N} 3-\mathrm{C} 6$ & $1.3506(18)$ & $\mathrm{C} 9-\mathrm{H} 9 \mathrm{~B}$ & $0.95(3)$ \\
$\mathrm{C} 3-\mathrm{H} 3$ & $0.99(2)$ & $\mathrm{C} 9-\mathrm{H} 9 \mathrm{C}$ & $0.98(3)$ \\
$\mathrm{C} 3-\mathrm{C} 4$ & $1.395(2)$ & $\mathrm{C} 10-\mathrm{H} 10$ & $0.965(17)$ \\
$\mathrm{N} 4-\mathrm{C} 8$ & $1.3278(18)$ & $\mathrm{C} 10-\mathrm{C} 11$ & $1.361(2)$
\end{tabular}




\begin{tabular}{|c|c|c|c|}
\hline $\mathrm{C} 4-\mathrm{C} 5$ & $1.492(2)$ & C11-H11 & $0.941(18)$ \\
\hline N5-N6 & $1.3591(15)$ & $\mathrm{C} 11-\mathrm{C} 12$ & $1.401(2)$ \\
\hline $\mathrm{N} 5-\mathrm{C} 10$ & $1.3490(18)$ & $\mathrm{C} 12-\mathrm{C} 13$ & $1.488(2)$ \\
\hline $\mathrm{C} 5-\mathrm{H} 5 \mathrm{~A}$ & $1.045(13)$ & $\mathrm{C} 13-\mathrm{H} 13 \mathrm{~A}$ & $0.98(3)$ \\
\hline $\mathrm{C} 5-\mathrm{H} 5 \mathrm{~B}$ & $1.045(13)$ & $\mathrm{C} 13-\mathrm{H} 13 \mathrm{~B}$ & $0.92(3)$ \\
\hline $\mathrm{C} 5-\mathrm{H} 5 \mathrm{C}$ & $1.045(13)$ & $\mathrm{C} 13-\mathrm{H} 13 \mathrm{C}$ & $0.95(3)$ \\
\hline $\mathrm{N} 2-\mathrm{N} 1-\mathrm{C} 1$ & $121.52(11)$ & $\mathrm{H} 5 \mathrm{~B}-\mathrm{C} 5-\mathrm{H} 5 \mathrm{E}$ & 141.1 \\
\hline $\mathrm{C} 2-\mathrm{N} 1-\mathrm{C} 1$ & $125.94(12)$ & $\mathrm{H} 5 \mathrm{~B}-\mathrm{C} 5-\mathrm{H} 5 \mathrm{~F}$ & 56.3 \\
\hline $\mathrm{C} 2-\mathrm{N} 1-\mathrm{N} 2$ & $112.52(12)$ & $\mathrm{H} 5 \mathrm{C}-\mathrm{C} 5-\mathrm{H} 5 \mathrm{D}$ & 56.3 \\
\hline $\mathrm{N} 1-\mathrm{C} 1-\mathrm{H} 1$ & $107.0(9)$ & $\mathrm{H} 5 \mathrm{C}-\mathrm{C} 5-\mathrm{H} 5 \mathrm{E}$ & 56.3 \\
\hline $\mathrm{N} 1-\mathrm{C} 1-\mathrm{N} 3$ & $111.06(11)$ & $\mathrm{H} 5 \mathrm{C}-\mathrm{C} 5-\mathrm{H} 5 \mathrm{~F}$ & 141.1 \\
\hline $\mathrm{N} 1-\mathrm{C} 1-\mathrm{N} 5$ & $111.55(11)$ & $\mathrm{H} 5 \mathrm{D}-\mathrm{C} 5-\mathrm{H} 5 \mathrm{E}$ & 109.5 \\
\hline $\mathrm{N} 3-\mathrm{C} 1-\mathrm{H} 1$ & $108.3(9)$ & $\mathrm{H} 5 \mathrm{D}-\mathrm{C} 5-\mathrm{H} 5 \mathrm{~F}$ & 109.5 \\
\hline $\mathrm{N} 3-\mathrm{C} 1-\mathrm{N} 5$ & $111.27(11)$ & $\mathrm{H} 5 \mathrm{E}-\mathrm{C} 5-\mathrm{H} 5 \mathrm{~F}$ & 109.5 \\
\hline $\mathrm{N} 5-\mathrm{C} 1-\mathrm{H} 1$ & $107.4(9)$ & $\mathrm{C} 12-\mathrm{N} 6-\mathrm{N} 5$ & $104.82(11)$ \\
\hline $\mathrm{C} 4-\mathrm{N} 2-\mathrm{N} 1$ & $104.33(12)$ & $\mathrm{N} 3-\mathrm{C} 6-\mathrm{H} 6$ & $120.6(10)$ \\
\hline $\mathrm{N} 1-\mathrm{C} 2-\mathrm{H} 2$ & $121.4(11)$ & $\mathrm{N} 3-\mathrm{C} 6-\mathrm{C} 7$ & $106.53(13)$ \\
\hline $\mathrm{N} 1-\mathrm{C} 2-\mathrm{C} 3$ & $106.24(15)$ & $\mathrm{C} 7-\mathrm{C} 6-\mathrm{H} 6$ & $132.8(10)$ \\
\hline $\mathrm{C} 3-\mathrm{C} 2-\mathrm{H} 2$ & $132.4(11)$ & $\mathrm{C} 6-\mathrm{C} 7-\mathrm{H} 7$ & $127.0(11)$ \\
\hline $\mathrm{N} 4-\mathrm{N} 3-\mathrm{C} 1$ & $117.37(11)$ & $\mathrm{C} 6-\mathrm{C} 7-\mathrm{C} 8$ & $105.75(13)$ \\
\hline $\mathrm{C} 6-\mathrm{N} 3-\mathrm{C} 1$ & $130.02(12)$ & $\mathrm{C} 8-\mathrm{C} 7-\mathrm{H} 7$ & $127.2(11)$ \\
\hline $\mathrm{C} 6-\mathrm{N} 3-\mathrm{N} 4$ & $112.10(11)$ & $\mathrm{N} 4-\mathrm{C} 8-\mathrm{C} 7$ & $111.04(13)$ \\
\hline $\mathrm{C} 2-\mathrm{C} 3-\mathrm{H} 3$ & $126.0(13)$ & $\mathrm{N} 4-\mathrm{C} 8-\mathrm{C} 9$ & $120.42(14)$ \\
\hline $\mathrm{C} 2-\mathrm{C} 3-\mathrm{C} 4$ & $106.23(15)$ & $\mathrm{C} 7-\mathrm{C} 8-\mathrm{C} 9$ & $128.53(14)$ \\
\hline $\mathrm{C} 4-\mathrm{C} 3-\mathrm{H} 3$ & $127.8(13)$ & $\mathrm{C} 8-\mathrm{C} 9-\mathrm{H} 9 \mathrm{~A}$ & $110.7(14)$ \\
\hline $\mathrm{C} 8-\mathrm{N} 4-\mathrm{N} 3$ & $104.56(11)$ & $\mathrm{C} 8-\mathrm{C} 9-\mathrm{H} 9 \mathrm{~B}$ & $110.7(15)$ \\
\hline $\mathrm{N} 2-\mathrm{C} 4-\mathrm{C} 3$ & $110.68(14)$ & $\mathrm{C} 8-\mathrm{C} 9-\mathrm{H} 9 \mathrm{C}$ & $109.5(15)$ \\
\hline $\mathrm{N} 2-\mathrm{C} 4-\mathrm{C} 5$ & $120.61(16)$ & $\mathrm{H} 9 \mathrm{~A}-\mathrm{C} 9-\mathrm{H} 9 \mathrm{~B}$ & $113(2)$ \\
\hline $\mathrm{C} 3-\mathrm{C} 4-\mathrm{C} 5$ & $128.69(16)$ & $\mathrm{H} 9 \mathrm{~A}-\mathrm{C} 9-\mathrm{H} 9 \mathrm{C}$ & $104.5(19)$ \\
\hline $\mathrm{N} 6-\mathrm{N} 5-\mathrm{C} 1$ & $117.49(11)$ & $\mathrm{H} 9 \mathrm{~B}-\mathrm{C} 9-\mathrm{H} 9 \mathrm{C}$ & $108(2)$ \\
\hline $\mathrm{C} 10-\mathrm{N} 5-\mathrm{C} 1$ & $130.21(12)$ & $\mathrm{N} 5-\mathrm{C} 10-\mathrm{H} 10$ & $120.1(10)$ \\
\hline $\mathrm{C} 10-\mathrm{N} 5-\mathrm{N} 6$ & $111.75(11)$ & $\mathrm{N} 5-\mathrm{C} 10-\mathrm{C} 11$ & $106.96(13)$ \\
\hline $\mathrm{C} 4-\mathrm{C} 5-\mathrm{H} 5 \mathrm{~A}$ & 109.5 & $\mathrm{C} 11-\mathrm{C} 10-\mathrm{H} 10$ & $132.9(10)$ \\
\hline $\mathrm{C} 4-\mathrm{C} 5-\mathrm{H} 5 \mathrm{~B}$ & 109.5 & $\mathrm{C} 10-\mathrm{C} 11-\mathrm{H} 11$ & $127.0(10)$ \\
\hline $\mathrm{C} 4-\mathrm{C} 5-\mathrm{H} 5 \mathrm{C}$ & 109.5 & $\mathrm{C} 10-\mathrm{C} 11-\mathrm{C} 12$ & $105.53(13)$ \\
\hline $\mathrm{C} 4-\mathrm{C} 5-\mathrm{H} 5 \mathrm{D}$ & 109.5 & $\mathrm{C} 12-\mathrm{C} 11-\mathrm{H} 11$ & $127.5(10)$ \\
\hline $\mathrm{C} 4-\mathrm{C} 5-\mathrm{H} 5 \mathrm{E}$ & 109.5 & $\mathrm{~N} 6-\mathrm{C} 12-\mathrm{C} 11$ & $110.93(13)$ \\
\hline $\mathrm{C} 4-\mathrm{C} 5-\mathrm{H} 5 \mathrm{~F}$ & 109.5 & $\mathrm{~N} 6-\mathrm{C} 12-\mathrm{C} 13$ & $120.15(14)$ \\
\hline $\mathrm{H} 5 \mathrm{~A}-\mathrm{C} 5-\mathrm{H} 5 \mathrm{~B}$ & 109.5 & $\mathrm{C} 11-\mathrm{C} 12-\mathrm{C} 13$ & $128.92(15)$ \\
\hline $\mathrm{H} 5 \mathrm{~A}-\mathrm{C} 5-\mathrm{H} 5 \mathrm{C}$ & 109.5 & $\mathrm{C} 12-\mathrm{C} 13-\mathrm{H} 13 \mathrm{~A}$ & $112.2(15)$ \\
\hline $\mathrm{H} 5 \mathrm{~A}-\mathrm{C} 5-\mathrm{H} 5 \mathrm{D}$ & 141.1 & $\mathrm{C} 12-\mathrm{C} 13-\mathrm{H} 13 \mathrm{~B}$ & $110.5(16)$ \\
\hline $\mathrm{H} 5 \mathrm{~A}-\mathrm{C} 5-\mathrm{H} 5 \mathrm{E}$ & 56.3 & $\mathrm{C} 12-\mathrm{C} 13-\mathrm{H} 13 \mathrm{C}$ & $108.8(16)$ \\
\hline $\mathrm{H} 5 \mathrm{~A}-\mathrm{C} 5-\mathrm{H} 5 \mathrm{~F}$ & 56.3 & $\mathrm{H} 13 \mathrm{~A}-\mathrm{C} 13-\mathrm{H} 13 \mathrm{~B}$ & $112(2)$ \\
\hline $\mathrm{H} 5 \mathrm{~B}-\mathrm{C} 5-\mathrm{H} 5 \mathrm{C}$ & 109.5 & $\mathrm{H} 13 \mathrm{~A}-\mathrm{C} 13-\mathrm{H} 13 \mathrm{C}$ & $107(2)$ \\
\hline $\mathrm{H} 5 \mathrm{~B}-\mathrm{C} 5-\mathrm{H} 5 \mathrm{D}$ & 56.3 & $\mathrm{H} 13 \mathrm{~B}-\mathrm{C} 13-\mathrm{H} 13 \mathrm{C}$ & $106(2)$ \\
\hline $\mathrm{N} 1-\mathrm{C} 1-\mathrm{N} 3-\mathrm{N} 4$ & $145.25(12)$ & $\mathrm{C} 2-\mathrm{C} 3-\mathrm{C} 4-\mathrm{C} 5$ & $178.91(17)$ \\
\hline
\end{tabular}




$\begin{array}{llll}\mathrm{N} 1-\mathrm{C} 1-\mathrm{N} 3-\mathrm{C} 6 & -43.68(19) & \mathrm{N} 3-\mathrm{C} 1-\mathrm{N} 5-\mathrm{N} 6 & 142.82(11) \\ \mathrm{N} 1-\mathrm{C} 1-\mathrm{N} 5-\mathrm{N} 6 & -92.55(13) & \mathrm{N} 3-\mathrm{C} 1-\mathrm{N} 5-\mathrm{C} 10 & -46.46(19) \\ \mathrm{N} 1-\mathrm{C} 1-\mathrm{N} 5-\mathrm{C} 10 & 78.17(17) & \mathrm{N} 3-\mathrm{N} 4-\mathrm{C} 8-\mathrm{C} 7 & -0.73(16) \\ \mathrm{N} 1-\mathrm{N} 2-\mathrm{C} 4-\mathrm{C} 3 & -0.18(17) & \mathrm{N} 3-\mathrm{N} 4-\mathrm{C} 8-\mathrm{C} 9 & 179.43(15) \\ \mathrm{N} 1-\mathrm{N} 2-\mathrm{C} 4-\mathrm{C} 5 & -179.06(15) & \mathrm{N} 3-\mathrm{C} 6-\mathrm{C} 7-\mathrm{C} 8 & 0.29(18) \\ \mathrm{N} 1-\mathrm{C} 2-\mathrm{C} 3-\mathrm{C} 4 & -0.05(19) & \mathrm{N} 4-\mathrm{N} 3-\mathrm{C} 6-\mathrm{C} 7 & -0.78(17) \\ \mathrm{C} 1-\mathrm{N} 1-\mathrm{N} 2-\mathrm{C} 4 & -178.37(12) & \mathrm{N} 5-\mathrm{C} 1-\mathrm{N} 3-\mathrm{N} 4 & -89.84(14) \\ \mathrm{C} 1-\mathrm{N} 1-\mathrm{C} 2-\mathrm{C} 3 & 178.37(14) & \mathrm{N} 5-\mathrm{C} 1-\mathrm{N} 3-\mathrm{C} 6 & 81.22(18) \\ \mathrm{C} 1-\mathrm{N} 3-\mathrm{N} 4-\mathrm{C} 8 & 173.56(12) & \mathrm{N} 5-\mathrm{N} 6-\mathrm{C} 12-\mathrm{C} 11 & -0.56(15) \\ \mathrm{C} 1-\mathrm{N} 3-\mathrm{C} 6-\mathrm{C} 7 & -172.22(14) & \mathrm{N} 5-\mathrm{N} 6-\mathrm{C} 12-\mathrm{C} 13 & 180.00(14) \\ \mathrm{C} 1-\mathrm{N} 5-\mathrm{N} 6-\mathrm{C} 12 & 173.41(11) & \mathrm{N} 5-\mathrm{C} 10-\mathrm{C} 11-\mathrm{C} 12 & 0.69(16) \\ \mathrm{C} 1-\mathrm{N} 5-\mathrm{C} 10-\mathrm{C} 11 & -172.24(13) & \mathrm{N} 6-\mathrm{N} 5-\mathrm{C} 10-\mathrm{C} 11 & -1.10(16) \\ \mathrm{N} 2-\mathrm{N} 1-\mathrm{C} 1-\mathrm{N} 3 & 71.12(16) & \mathrm{C} 6-\mathrm{N} 3-\mathrm{N} 4-\mathrm{C} 8 & 0.94(16) \\ \mathrm{N} 2-\mathrm{N} 1-\mathrm{C} 1-\mathrm{N} 5 & -53.63(16) & \mathrm{C} 6-\mathrm{C} 7-\mathrm{C} 8-\mathrm{N} 4 & 0.29(18) \\ \mathrm{N} 2-\mathrm{N} 1-\mathrm{C} 2-\mathrm{C} 3 & -0.06(18) & \mathrm{C} 6-\mathrm{C} 7-\mathrm{C} 8-\mathrm{C} 9 & -179.89(18) \\ \mathrm{C} 2-\mathrm{N} 1-\mathrm{C} 1-\mathrm{N} 3 & -107.19(16) & \mathrm{C} 10-\mathrm{N} 5-\mathrm{N} 6-\mathrm{C} 12 & 1.03(15) \\ \mathrm{C} 2-\mathrm{N} 1-\mathrm{C} 1-\mathrm{N} 5 & 128.06(15) & \mathrm{C} 10-\mathrm{C} 11-\mathrm{C} 12-\mathrm{N} 6 & -0.07(17) \\ \mathrm{C} 2-\mathrm{N} 1-\mathrm{N} 2-\mathrm{C} 4 & 0.15(16) & \mathrm{C} 10-\mathrm{C} 11-\mathrm{C} 12-\mathrm{C} 13 & 179.30(17) \\ \mathrm{C} 2-\mathrm{C} 3-\mathrm{C} 4-\mathrm{N} 2 & 0.1(2) & & \end{array}$

Hydrogen-bond geometry $\left(A,{ }^{\circ}\right)$

\begin{tabular}{lllll}
\hline$D-\mathrm{H} \cdots A$ & $D-\mathrm{H}$ & $\mathrm{H} \cdots A$ & $D \cdots A$ & $D-\mathrm{H} \cdots A$ \\
\hline $\mathrm{C} 1-\mathrm{H} 1 \cdots \mathrm{N} 6 \mathrm{i}$ & $0.96(2)$ & $2.44(2)$ & $3.3796(19)$ & $167(1)$ \\
$\mathrm{C} 6-\mathrm{H} 6 \cdots \mathrm{N} 2$ & $0.932(17)$ & $2.529(16)$ & $3.0918(19)$ & $119.2(13)$ \\
\hline
\end{tabular}

Symmetry code: (i) $-x+2,-y+1,-z+2$. 